\title{
Selective separation of polychlorinated naphthalene (PCNs), hexabromocyclododecanes (HBCDs) and tetrabromobisphenol A (TBBPA) in soil matrices
}

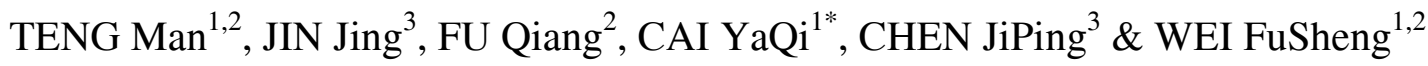 \\ ${ }^{1}$ Research Center for Eco-Environmental Sciences, Chinese Academy of Sciences, Beijing 100085, China; \\ ${ }^{2}$ China National Environmental Monitoring Center, Beijing 100012, China; \\ ${ }^{3}$ Dalian Institute of Chemical Physics, Chinese Academy of Sciences, Dalian 116023, China
}

Received April 10, 2012; accepted June 5, 2012

\begin{abstract}
A method for the selective separation of polychlorinated naphthalene (PCNs), hexabromocyclododecanes (HBCDs) and tetrabromobisphenol A (TBBPA) in complex matrices was developed. In the present study, the type of sorbents and the volume and composition of eluting solvents were investigated in detail. Under the optimal conditions, the recoveries of PCNs, HBCDs and TBBPA in different fractions were determined with liquid/gas chromatography tandem mass spectrometry (LC or GC-MS/MS), which was nearly close to $100 \%$. To investigate the feasibility of the developed method, it was applied to isolate target compounds from spiked soil extracts. PCNs, HBCDs and TBBPA could be subsequently eluted with hexane, hexane/dichloromethane $(1: 1)$, and hexane/dichloromethane $(1: 3)$, and the recoveries of the analysts from three fractions were satisfactory $(70.9 \%-107 \%)$ with the exception of $\gamma$-HBCD at low levels. The results indicated that the method exhibited good performance for the selective separation of PCNs, HBCDs and TBBPA and showed great potential in sample pretreatment in terms of alleviating influence from different class of impurities to some extent.
\end{abstract}

hexabromocyclododecane, polychlorinated naphthalene, selective separation, tetrabromobisphenol A

Citation: Teng M, Jin J, Fu Q, et al. Selective separation of polychlorinated naphthalene (PCNs), hexabromocyclododecanes (HBCDs) and tetrabromobisphenol A (TBBPA) in soil matrices. Chin Sci Bull, 2013, 58: 500-506, doi: 10.1007/s11434-012-5638-5

Flame retardants (FRs) are chemicals used in thermoplastics, thermosets, wood, paper, electronics, textiles and coatings to meet fire safety regulations. In general, FRs consist of several different classes of chemicals, including minerals, organohalogen compounds, and organophosphorus compounds. As one of the most important class, organohalogen FRs are produced in significant amount. Hereinto, brominated flame retardants (BFRs) and chlorinated flame retardants (CFRs) are relatively new generations of FRs in many types of consumer products. The last few decades have seen dramatic growth in the scale of production and usage. Not surprisingly, these chemicals attract much attention in environmental field, owing to their high production volumes and

*Corresponding author (email: caiyaqi@rcees.ac.cn) persistent organic pollutants (POPs) character, such as tetrabromobisphenol A (TBBPA), hexabromocyclododecanes (HBCDs) and polychlorinated naphthalene (PCNs).

These chemicals have been detected in various matrices, including air [1-3], soil [4,5], sediment [6,7], sewage sludge [8,9], water [10-12] and human [13]. They are considered harmful, having been linked to liver, thyroid, reproductive/ developmental, and neurological effects [14]. At present, the risk assessment of HBCDs has been finished, and it has been listed as a POPs candidate, indicating that its production will be limited or prohibited in future. TBBPA is the largest brominated flame retardant in terms of production volume with annual global consumption amounting to $120000 \mathrm{t}$ [15]. The potential harm of TBBPA to human and environment needs further investigation. Additionally, pol- 
ychlorinated naphthalene (PCNs), as one class of CFRs, has been the hot topic discussed in the seventh meeting of the Persistent Organic Pollutants Review Committee (POPRC). Therefore, it is essential for analysts to study their sources, distribution, levels, and toxicity, which means that the analysis of PCNs, HBCDs and TBBPA in environmental samples is exigent.

Due to the excellent separation capacity for congeners, gas chromatography coupled with mass spectrometry (GCMS) is a primary technique for PCNs analysis. This technique is also applied to the analysis of HBCDs, but it is proved to be non-isomer-specific because the diastereomers inter-convert at temperatures over $160^{\circ} \mathrm{C}$ and decompose at temperatures above $240^{\circ} \mathrm{C}[16,17]$. In addition, the partition of HBCDs on GC column stationary phases is poor [18]. Therefore, isomer-specific method based on liquid chromatography/tandem mass spectrometry has been reported for HBCDs analysis, which has been proved to be an important advancement in the area of HBCDs research due to the highlighted differences in the environmental fate and behavior among the diastereoisomers [2,19,20]. The LC-MS/ MS method has been routinely applied for the analysis of TBBPA because of the advantages of avoiding derivatization prior to GC analysis. For TBBPA, signal intensity improvements gained using quadrupole and ion-trap mass spectrometry in different ionization modes such as atmospheric pressure chemical ionization (APCI) and electrospray ionization (ESI) have been discussed previously [21,22]. However, LC-ESI-MS analysis was prone to ion suppression resulting in decreased sensitivity [23]. Therefore, it is exigent to develop a method to remove as many impurities as possible prior to LC/GC-MS analysis. Simultaneous analysis of HBCDs and TBBPA has been reported in literature $[8,24]$. HBCDs and TBBPA can be separated with various sorbents (e.g., silica, florisil and Oasis HLB) by elution with different solvents (e.g., the mixture of hexane and dichloromethane or acetone for HBCDs, dichloromethane for TBBPA) $[21,25,26]$. But there are no reports about the simultaneous analysis of PCNs, HBCDs and TBBPA by far. The study aims for developing a selective separation method to perform the simultaneous analysis of PCNs, HBCDs and TBBPA. Meanwhile, it is requested to alleviate matrixrelated effects to some extent.

\section{Experimental}

\subsection{Chemical and materials}

Methanol (MeOH) (HPLC grade) and acetonitrile (ACN) (HPLC grade) were supplied by Fisher (Fair Lawn, NJ, USA). Dichloromethane (DCM), n-hexane and acetone were all for organic residue analysis and they were purchased from J.T. Baker (Phillipsburg, NJ, USA). Silica was purchased from MP Biomedicals (Eschwege, Germany). PCNs $\left(5 \mu \mathrm{g} \mathrm{mL}^{-1}\right)$, TBBPA $\left(50 \mu \mathrm{g} \mathrm{mL}^{-1}\right), \alpha-\mathrm{HBCD}$ and
$\gamma$-HBCD $\left(50 \mu \mathrm{g} \mathrm{mL}^{-1}\right)$ isomers were provided by Wellington Laboratories. Mixed series working standards of TBBPA, $\alpha-\mathrm{HBCD}, \gamma$-HBCD and PCNs were prepared by serial dilutions of these stock solutions with hexane.

\subsection{Rinsing program}

(1) Silica gel as sorbents. A total of $8 \mathrm{~g}$ of silica gel was packed into a column and compacted with a vacuum pump. Then $50 \mathrm{~mL}$ of hexane was used to wash silica gel. Next, $10 \mathrm{ng}$ of PCNs and $100 \mathrm{ng}$ of TBBPA and HBCDs standard solutions were loaded. Target compounds retained on silica gel were eluted subsequently with $200 \mathrm{~mL}$ of hexane (f1), $200 \mathrm{~mL}$ of hexane/DCM (1:1) (f2), and $4 \times 50 \mathrm{~mL}$ of DCM (f3-f6). These fractions (f1-f6) were concentrated nearly to $500 \mu \mathrm{L}$, and then transferred to cuvette, respectively. Then washing solvents for each corresponding flask were also transferred to cuvette. After solvent combination, they were evaporated to dryness and reconstituted to $100 \mu \mathrm{L}$ with hexane for fraction $\mathrm{f} 1$ and $500 \mu \mathrm{L}$ with methanol for fraction f2-f6.

(2) $3 \%$ deactivated silica gel as sorbents. Prior to packing, silica gel was activated at $130^{\circ} \mathrm{C}$ for $15 \mathrm{~h}$, and then cooled to room temperature in a dessicator. After that, $3 \%$ $(\mathrm{w} / \mathrm{w})$ ultrapure water was sprinkled on silica gel followed by blending with a shaker. The prepared sorbents was stored in a pre-cleaned bottle with screw cap and then placed in the dessicator. The following experiment was performed according to the description mentioned in section 2.2.1 except that target compounds were eluted with $100 \mathrm{~mL}$ of hexane (f1'), $2 \times 50 \mathrm{~mL}$ of hexane/DCM (1:1) (f2'-f3'), $2 \times 50 \mathrm{~mL}$ of hexane/DCM $(1: 3)\left(\mathrm{f} 4^{\prime}-\mathrm{f} 5^{\prime}\right)$ and $2 \times 25 \mathrm{~mL}$ of DCM (f6'f7'), respectively.

\subsection{Pretreatment of real samples}

(1) Soxhlet extraction. Soil sample was collected from Mianyang suburb in Sichuan province and freeze-dried for $24 \mathrm{~h}$ with Labconco freeze dry system and then ground into powders followed by passing through a 60-mesh testing sieve. Next, $10 \mathrm{~g}$ soil sample was subjected to soxhlet extraction with $250 \mathrm{~mL}$ of hexane/DCM $(1: 1)$. The raw extract was concentrated to approximate $500 \mu \mathrm{L}$ using rotary evaporation and transferred into a cuvette. After rinsing the concentration vessel with DCM three times, these two fractions were combined.

(2) Lipid and sulphur removal. To remove lipids and sulphur, the obtained extract was subjected to gel permeation chromatography (GPC) using a P230 high pressure and constant-flow pump and a cross-linked vinylbenzene-styrene copolymer (SX-3 Bio-Beads or equivalent) packed column (ID/length $25 \mathrm{~mm} \times 450 \mathrm{~mm}$ ). DCM was used as moblie phase at a flow rate of $5 \mathrm{~mL} \mathrm{~min}$. The data were obtained by Biotronik UV detector BT 3030 at a wavelength of 
$254 \mathrm{~nm}$. The fraction in the time range of 21-41 min was collected and evaporated to dryness followed by reconstitution with $3 \mathrm{~mL}$ of hexane.

(3) Selective separation of spiked soil extract. A total of $200 \mu \mathrm{L}$ of the above-mentioned soil extract was spiked with two levels of PCNs, HBCDs and TBBPA standard (level 1, $10 \mathrm{ng}$ of PCNs, $100 \mathrm{ng}$ of HBCDs and TBBPA; level 2, $4 \mathrm{ng}$ of PCNs, $40 \mathrm{ng}$ of HBCDs and TBBPA). Then, selective separation was performed using 3\% deactivated silica gel packed column. PCNs, HBCDs and TBBPA were eluted subsequently with $100 \mathrm{~mL}$ of hexane, $100 \mathrm{~mL}$ of hexane/DCM $(1: 1)$ and $100 \mathrm{~mL}$ hexane/DCM $(1: 3)$. Each fraction was evaporated to dryness and reconstituted to 100 $\mu \mathrm{L}$ with hexane for PCNs and $500 \mu \mathrm{L}$ with methanol for HBCDs and TBBPA.

\subsection{Instrumental analysis}

For PCNs, pretreated samples were analyzed on TSQ Quantum XLS Thermo Fisher GC-MS/MS equipment. GC separation was performed on a $30 \mathrm{~m}$ TR-5MS silica capillary column (i.d. $0.25 \mathrm{~mm}$, film thickness $0.25 \mu \mathrm{m}$; Thermo Fisher Scientific) operated with helium as carrier gas at 1.0 $\mathrm{mL} \min ^{-1}$. Sample injection was splitless, followed by opening split after $1 \mathrm{~min}$ with the split flow of $50 \mathrm{~mL} \mathrm{~min}^{-1}$. The oven temperature programmer was: $100^{\circ} \mathrm{C}$ hold for $2 \mathrm{~min}$, $20^{\circ} \mathrm{C} \mathrm{m^{-1 }}$ to $200^{\circ} \mathrm{C}, 10^{\circ} \mathrm{C} \mathrm{min}^{-1}$ to $250^{\circ} \mathrm{C}$ hold for $15 \mathrm{~min}$. The temperature of both the inlet and MS transfer line was $260^{\circ} \mathrm{C}$ while the source temperature was $220^{\circ} \mathrm{C}$. The monitored masses for tetra-CNs, penta-CNs, hexa-CNs, hepta-CNs and octa-CNs were 266, 302, 336, 370 and 404, respectively.

For TBBPA and HBCDs, pretreated fractions were analyzed on TSQ Quantum Access Thermo Fisher LC-MS/MS instrument. LC separation was performed on an Atlantis T3 column $(2.1 \mathrm{~mm} \times 150 \mathrm{~mm}, 3 \mu \mathrm{m}$; Waters, Milford, Massachusetts, USA). The mobile phase was $\mathrm{MeOH} / \mathrm{ACN} / \mathrm{H}_{2} \mathrm{O}$ starting at $60 / 20 / 20(\mathrm{v} / \mathrm{v})$ with a flow rate of $200 \mu \mathrm{L}$. After $1 \mathrm{~min}$, the gradient was increased to $50 / 40 / 10(\mathrm{v} / \mathrm{v})$ in $8 \mathrm{~min}$, held for $6 \mathrm{~min}$, reversed back to 60/20/20 (v/v) in $1 \mathrm{~min}$, and then held for $15 \mathrm{~min}$. The ESI-MS/MS was operated in an electrospray negative ion mode; the optimized MS/MS parameters for TBBPA and HBCDs isomers were as follows: spray voltage, $-2800 \mathrm{~V}$; vaporizer temperature, $300^{\circ} \mathrm{C}$; sheath gas pressure, $30 \mathrm{psi}$; aux gas pressure, 5 psi; capillary temperature, $300^{\circ} \mathrm{C}$; collision energy, $-36--32 \mathrm{eV}$ for TBBPA with tube lens of 98 and $-45--55 \mathrm{eV}$ for HBCDs isomers with tube lens of 63 . Target compounds were determined by selective reaction monitoring (SRM). The ions were selected at the $\left([\mathrm{M}-\mathrm{H}]^{-}\right)$transition of $\mathrm{m} / \mathrm{z}$ $640.6 \rightarrow 79.3 / 640.6 \rightarrow 81.3$ for HBCDs isomers, and $\mathrm{m} / z$ $542.6 \rightarrow 447.7 / 542.6 \rightarrow 417.7 / 542.6 \rightarrow 290.5$ for TBBPA.

\section{Results and discussion}

Multi-layer silica gel column was commonly employed for the cleanup of sample matrices, including neutral silica gel, $\mathrm{H}_{2} \mathrm{SO}_{4}$ /silica gel, silver nitrate/silica gel, $\mathrm{KOH} /$ silica gel and anhydrous sodium sulfate [27,28]. This technique was also applied for PCNs analysis. But the application of $\mathrm{H}_{2} \mathrm{SO}_{4} /$ silica gel will decrease the recovery of TBBPA owing to the presence of active hydroxyl [29]. Considering about the irreversible adsorption of TBBPA on anhydrous sodium sulfate [30] and possible debromination of HBCDs on $\mathrm{KOH} /$ silica [31], neutral silica gel was selected as the predominate sorbents for cleanup in the present study. Its capability for the selective separation of PCNs, HBCDs and TBBPA was investigated systematically.

\subsection{Selective separation on silica gel column}

As a common sorbents for POPs analysis, silica was selected as sorbents for pretreatment of PCNs, HBCDs and TBBPA. The discrepancy of three compounds in $\log K_{\text {ow }}$ (4.5-8.2 for PCNs, 5.6 for HBCDs, 4.5 for TBBPA) [32-34] was beneficial to their selective separation [27].

As shown in Figure 1, target compounds retained on silica were eluted in sequence with different solvent. With
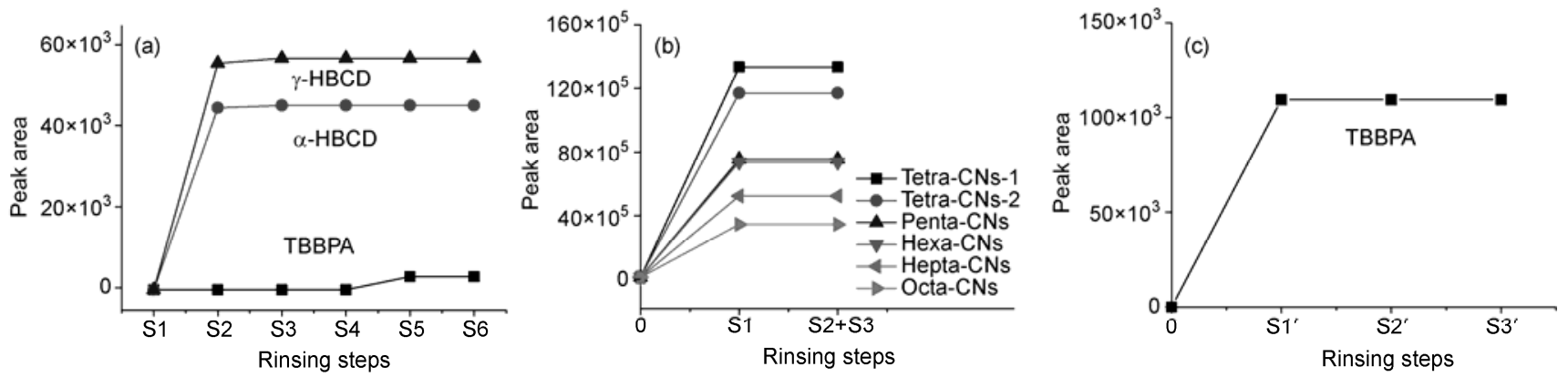

Figure 1 The elution of PCNs, HBCD and TBBPA from silica gel with different solvents. (a) HBCD and TBBPA detected from different fractions (f2-f6) using LC-MS-MS. (b) PCNs detected from fractions (f1-f3) using GC-MS. (c) TBBPA detected from eluants with the mixture of acetate acid and acetone $(1 \%, \mathrm{v} / \mathrm{v})$ as rinsing solvent. S1, $200 \mathrm{~mL}$ hexane, S2, $200 \mathrm{~mL}$ hexane/DCM (1:1), S3-S6, 4×50 mL DCM, S1'-S3', 3×100 mL the mixture of acetate acid and acetone $(1 \%, \mathrm{v} / \mathrm{v})$. 
$200 \mathrm{~mL}$ of hexane (S1 in Figure 1(b)), PCNs could be eluted completely; meanwhile the retention of HBCDs and TBBPA was not interfered (Figure 1(a)). With the continuance of rinsing step 2 (S2 in Figure 1(a)), HBCDs was collected thoroughly with $200 \mathrm{~mL}$ of hexane/DCM $(1: 1)$ without promoting the elution of PCNs and TBBPA (Figure 1(a) and (b)). Due to the presence of hydroxyl groups in the molecule structure of TBBPA, this compound showed strong retention on silica column. Even when the volume of DCM was increased to $200 \mathrm{~mL}$ (S3-S6 in Figure1(a)), TBBPA could not be eluted effectively from silica. These results agreed well with the fact that these compounds have different $K_{\text {ow }}$ values. Considering the intensive interaction between TBBPA and silica, acetate acid was added to rinsing solvents to weaken such retention interaction. As can be seen, TBBPA could be eluted from silica column with $100 \mathrm{~mL}$ of acetone containing $1 \%$ acetate acid (v/v, S1') (Figure 1(c)).

In general, the selective separation of PCNs, HBCDs and TBBPA could be performed with silica as sorbents. But there are two drawbacks to note. First, excess solvent (500 $\mathrm{mL}$ ) was consumed to elute analytes. Second, the evaporation of organic solvent became difficult due to the presence of acetate acid, and the color of solvent got colorful at last. Therefore, it was necessary to modify silica sorbents.

\subsection{Selective separation on $3 \%$ deactivated silica gel column}

From the aforementioned results, it is recognized that the strong interaction between TBBPA and silica blocked the elution of TBBPA from silica. It became spontaneous to attempt to deactivate silica with polar compound, such as water, which had been applied in a previous report [5]. In this study, the concentration and amount of organic solvent was optimized to get high separation efficiency.

As can be seen in Figure 2, HBCDs and TBBPA were not detected in fraction $1^{\prime}$, facilitating their isolation from

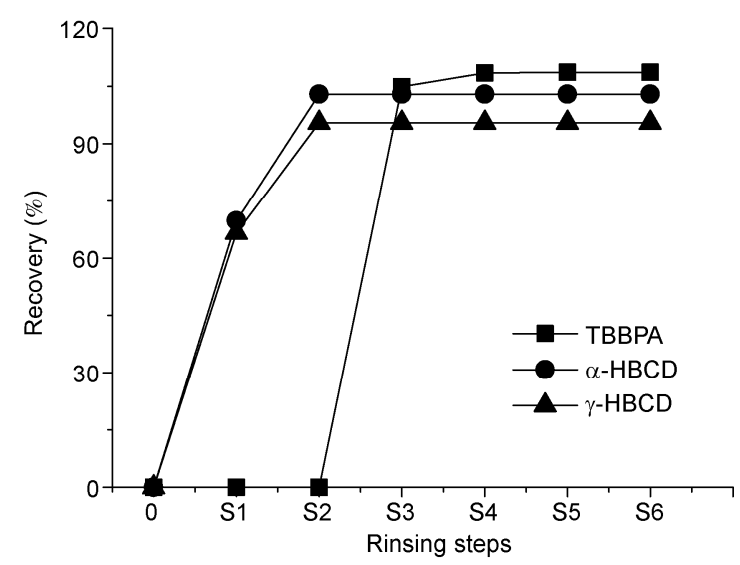

Figure 2 Recoveries of HBCD and TBBPA with different solvents on 3\% deactivated silica gel. S1, $100 \mathrm{~mL}$ of hexane, $\mathrm{S} 2-\mathrm{S} 3,2 \times 50 \mathrm{~mL}$ of hexane/DCM (1:1), S4-S5, 2×50 mL of hexane/DCM (1:3), S6-S7, 2× $50 \mathrm{~mL}$ DCM.
PCNs. The separation of HBCDs and TBBPA was achieved with $100 \mathrm{~mL}$ of hexane/DCM $(1: 1)\left(\mathrm{f} 2^{\prime}+\mathrm{f} 3^{\prime}\right)$. Most of TBBPA was eluted successfully with $100 \mathrm{~mL}$ of hexane/DCM $(1: 3)$ $\left(\mathrm{f} 4^{\prime}+\mathrm{f} 5^{\prime}\right)$, which overcame the hard desorption of this analyte on silica column. Then selective separation of PCNs, HBCDs and TBBPA was achieved with $3 \%$ deactivated silica gel. Generally, $3 \%$ deactivated silica gel was superior to silica for the selective separation of PCNs, HBCDs and TBBPA in terms of recovery, operation and solvent consumption. The selective separation of three compounds was controlled by adjusting the ratio of DCM and hexane in elution solution. Thus, 3\% deactivated silica gel was used as sorbents for the selective separation in the following experiments.

\subsection{Estimation of the method for the selective separation of PCNs, HBCDs and TBBPA}

According to the rinsing steps described in Section 2.2, mixed standard solution was loaded on $3 \%$ deactivated silica gel and eluted with $100 \mathrm{~mL}$ of hexane, $100 \mathrm{~mL}$ of hexane/DCM (1:1) and $100 \mathrm{~mL}$ of hexane/DCM (1:3) subsequently to collect PCNs, HBCDs and TBBPA. All experiments were conducted in triplicate, and average results were reported.

Figure 3 showed the selective ion monitoring (SIM) and selective reaction monitoring (SRM) chromatograms of different fractions, which proved that three groups of compounds could be detected in three fractions, respectively. As listed in Table 1, 86.1\%-101\% of PCNs was eluted with $100 \mathrm{~mL}$ hexane without detection of HBCDs and TBBPA. $105 \%$ of $\alpha$-HBCD and $\gamma$-HBCD were recovered in second fraction, and $107 \%$ of TBBPA was eluted in the last fraction. These recoveries were satisfactory with relative standard deviation (RSD) less than $9.0 \%$, indicating that the developed method was highly effective and selective.

\subsection{Feasibility of the selective separation method for application in soil extracts}

In order to investigate the feasibility of the proposed method, it was used to separate these three groups of compounds from spiked soil extract. Figure 3 showed the TIC chromatograms of each fraction. It can be seen that the developed method played an important role in providing clean fractions for analysis, alleviating interferences from impurities to some extent. After the selective separation, the recoveries of PCNs, HBCDs and TBBPA were summarized in Table 2. It was clear that the recoveries of TBBPA $(84.5 \%-87.0 \%)$ and $\alpha$-HBCD $(91.1 \%-92.3 \%)$ changed slightly with the spiked level, while that of PCNs decreased or increased to some extent depending on their molecular weight. Most importantly, the recovery of $\gamma$-HBCD decreased sharply from $87.4 \%$ to $58.3 \%$, when spiked level changed from 100 to $40 \mathrm{ng}$. In other words, the matrix had no significant effect 

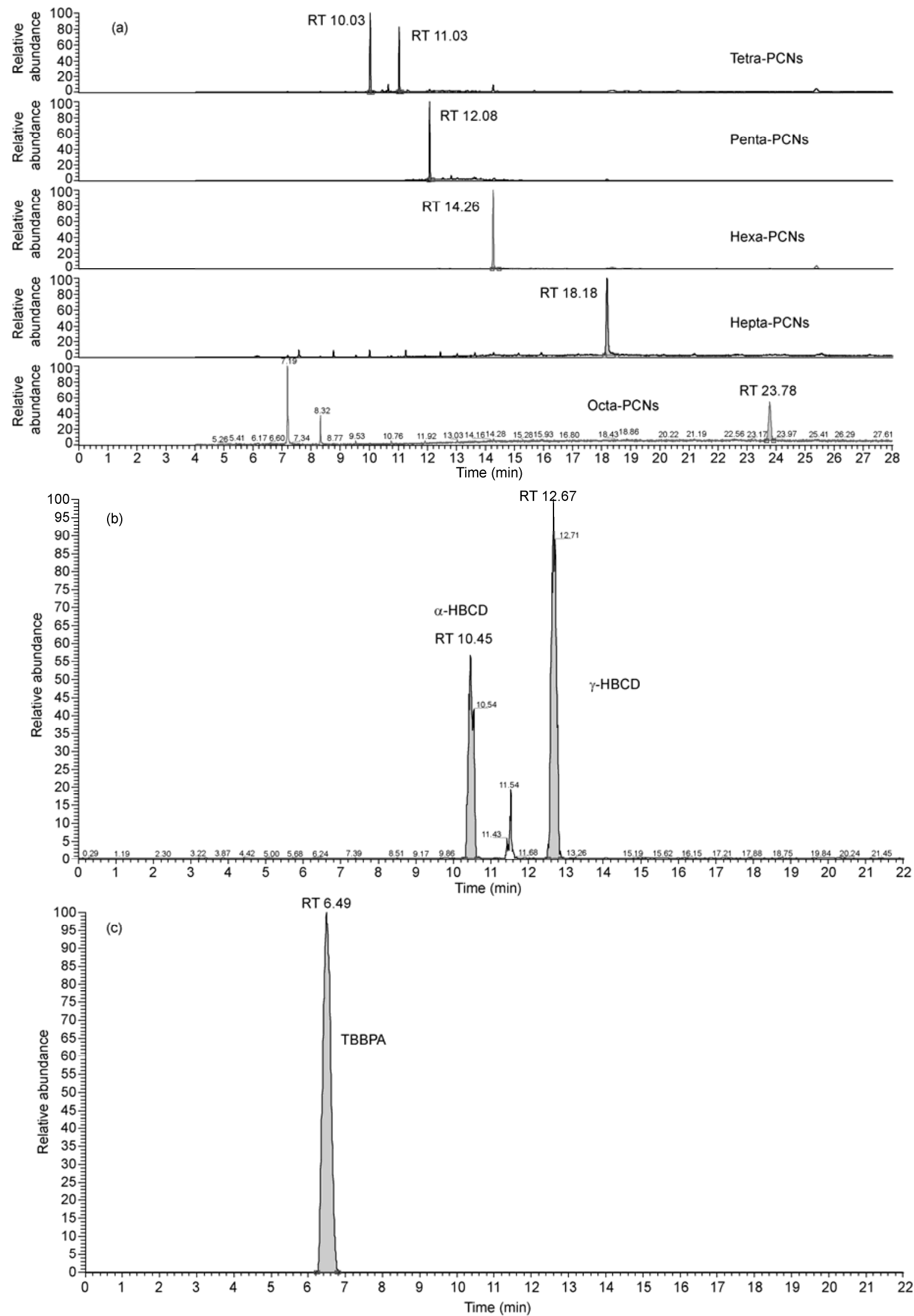

Figure 3 Selective ion monitoring (SIM) chromatogram of fraction A (100 mL of hexane), and selective reaction monitoring (SRM) chromatograms of fraction $\mathrm{B}(100 \mathrm{~mL}$ of hexane/DCM $(1: 1))$ and $\mathrm{C}(100 \mathrm{~mL}$ of hexane/DCM $(1: 3))$, respectively. 
Table 1 Recoveries of standard target compounds obtained from different rinsing steps ${ }^{\text {a) }}$

\begin{tabular}{|c|c|c|c|c|c|c|c|c|c|}
\hline \multirow{3}{*}{ Rinsing steps } & \multicolumn{9}{|c|}{ Recovery \pm relative standard deviation (\%) } \\
\hline & \multicolumn{6}{|c|}{10 ng PCNs } & \multicolumn{2}{|c|}{100 ng HBCD } & \multirow{2}{*}{100 ng TBBPA } \\
\hline & Tetra-1 & Tetra-2 & Penta- & Hexa- & Hepta- & Otca- & $\alpha-$ & $\gamma-$ & \\
\hline Step A & $86.1 \pm 1.4$ & $87.3 \pm 2.8$ & $101 \pm 5.0$ & $97.4 \pm 8.8$ & $97.8 \pm 3.8$ & $98.4 \pm 8.1$ & 0 & 0 & 0 \\
\hline Step B & - & - & - & - & - & - & $105 \pm 3.2$ & $105 \pm 7.7$ & 0 \\
\hline Step C & - & - & - & - & - & - & 0 & 0 & $107 \pm 2.4$ \\
\hline
\end{tabular}

a) Step A, $100 \mathrm{~mL}$ of hexane; Step B, $100 \mathrm{~mL}$ of hexane/DCM=1:1; Step C, $100 \mathrm{~mL}$ of hexane/DCM=1:3.

Table 2 Recoveries of target compounds in spiked soil extracts using selective separation method ${ }^{\text {a) }}$

\begin{tabular}{|c|c|c|c|c|c|c|c|c|c|}
\hline \multirow{3}{*}{ Rinsing steps } & \multicolumn{9}{|c|}{ Recovery \pm standard deviation (SD) (\%) } \\
\hline & \multicolumn{6}{|c|}{10 ng PCNs } & \multicolumn{2}{|c|}{100 ng HBCD } & \multirow{2}{*}{100 ng ТВBPA } \\
\hline & Tetra-1 & Tetra-2 & Penta & Hexa & Hepta & Octa & $\alpha-$ & $\gamma-$ & \\
\hline Step A & $83.4 \pm 6.3$ & $85.1 \pm 5.7$ & $84.1 \pm 3.7$ & $85.0 \pm 1.5$ & $88.4 \pm 3.4$ & $91.3 \pm 6.7$ & - & - & - \\
\hline Step B & - & - & - & - & - & & $91.1 \pm 4.6$ & $87.4 \pm 7.9$ & - \\
\hline Step C & - & - & - & - & - & & - & - & $84.5 \pm 13.7$ \\
\hline \multirow{3}{*}{ Rinsing steps } & \multicolumn{9}{|c|}{ Recovery \pm standard deviation (SD) (\%) } \\
\hline & \multicolumn{6}{|c|}{4 ng PCNs } & \multicolumn{2}{|c|}{$\begin{array}{c}40 \mathrm{ng} \\
\mathrm{HBCD}\end{array}$} & $40 \mathrm{ng}$ \\
\hline & Tetra-1 & Tetra-2 & Penta & Hexa & Hepta & Octa & $\alpha-$ & $\gamma-$ & TBBPA \\
\hline Step A & $70.9 \pm 10.1$ & $73.8 \pm 10.1$ & $90.8 \pm 14.2$ & $99.8 \pm 15.1$ & $107 \pm 14.8$ & $93.4 \pm 6.4$ & - & - & - \\
\hline Step B & - & - & - & - & - & & $92.3 \pm 5.3$ & $58.3 \pm 6.6$ & - \\
\hline Step C & - & - & - & - & - & & - & - & $87.0 \pm 6.4$ \\
\hline
\end{tabular}

a) Step A, $100 \mathrm{~mL}$ hexane; Step B, $100 \mathrm{~mL}$ hexane/DCM=1:1; Step C, $100 \mathrm{~mL}$ hexane/DCM=1:3.

on its recovery when high level of HBCDs was spiked in soil extract.

\section{Conclusion}

A method for the selective separation of PCNs, HBCDs and TBBPA was developed in this study. PCNs, HBCDs and TBBPA were eluted successfully with $100 \mathrm{~mL}$ of hexane, $100 \mathrm{~mL}$ of hexane/DCM $(1: 1)$ and $100 \mathrm{~mL}$ of hexane/DCM $(1: 3)$, subsequently. Compared to reported methods for the analysis of PCNs, HBCDs and TBBPA, the developed method exhibited advantages in terms of recovery, selectivity. In order to validate the feasibility of the proposed method, it was applied to spiked soil extract. The results indicated that the influence of matrix effect on the separation efficiency is negligible when $10 \mathrm{ng}$ of PCNs and $100 \mathrm{ng}$ of HBCDs and TBBPA were spiked with soil extract. But such matrix effect would play an important role in decreasing the recover of $\gamma$-HBCD. Therefore, it was suggested that isotope dilution combined with the selective separation was preferable, representing a selective and the most exact method for the analysis of PCNs, HBCDs and TBBPA. At present, the related work is still in progress.
This work was supported by the Special Environmental Protection Foundation for Public Welfare Project (201209016) and the National High Technology Research and Development Program of China (2007AA061602).

1 Hu J, Jin J, Wang Y, et al. Levels of polybrominated diphenyl ethers and hexabromocyclododecane in the atmosphere and tree bark from Beijing, China. Chemosphere, 2011, 84: 355-360

2 Yu Z, Chen L, Maw B, et al. Diastereoisomer- and enantiomer-specific profiles of hexabromocyclododecane in the atmosphere of an urban city in South China. Environ Sci Technol, 2008, 42: 3996-4001

3 Mari M, Schuhmacher M, Feliubadalo J, et al. Air concentrations of PCDD/Fs, PCBs and PCNs using active and passive air samplers. Chemosphere, 2008, 70: 1637-1643

4 Li Y, Zhou Q, Wang Y, et al. Fate of tetrabromobisphenol A and hexabromocyclododecane brominated flame retardants in soil and uptake by plants. Chemosphere, 2010, 82: 204-209

5 Yu Z, Peng P A, Sheng G, et al. Determination of hexabromocyclododecane diastereoisomers in air and soil by liquid chromatography-electrospray tandem mass spectrometry. J Chromatogr A, 2008, 1190: 74-79

6 Sellstrom U, Kierkegaard A, De Wit C, et al. Polybrominated diphenyl ethers and hexabromocyclododecane in sediment and fish from a Swedish river. Environ Toxicol Chem, 1998, 17: 1065-1072

7 Pan X, Tang J, Chen Y, et al. Polychlorinated naphthalenes (PCNs) in riverine and marine sediments of the Laizhou Bay area, North China. Environ Pollut, 2011, 159: 3515-3521

8 Guerra P, Eljarrat E, Barcelo D. Simultaneous determination of hexabromocyclododecane, tetrabromobisphenol A, and related compounds in sewage sludge and sediment samples from Ebro River basin (Spain). 
Anal Bioanal Chem, 2010, 397: 2817-2824

9 Stevens J L, Northcott G L, Stern G A, et al. PAHs, PCBs, PCNs, organochlorine pesticides, synthetic musks, and polychlorinated $n$-alkanes in UK sewage sludge: Survey results and implications. Environ Sci Technol, 2003, 37: 462-467

10 Zhao R S, Hu C, Zhou J B, et al. Preconcentration and sensitive determination of hexabromocyclododecane diastereomers in environmental water samples using solid phase extraction with bamboo charcoal cartridge prior to rapid resolution liquid chromatographyelectrospray tandem mass spectrometry. Anal Bioanal Chem, 2011, 400: $1189-1195$

11 Zhao R S, Wang S S, Cheng C G, et al. Rapid enrichment and sensitive determination of tetrabromobisphenol A in environmental water samples with ionic liquid dispersive liquid-phase microextraction prior to HPLC-ESI-MS-MS. Chromatographia, 2011, 73: 793-797

12 Ishaq R, Persson N J, Zebuhr Y, et al. PCNs, PCDD/Fs, and NonorthoPCBs, in water and bottom sediments from the industrialized norwegian grenlandsfjords. Environ Sci Technol, 2009, 43: 34423447

13 Eljarrat E, Guerra P, Martinez E, et al. Hexabromocyclododecane in human breast milk: Levels and enantiomeric patterns. Environ Sci Technol, 2009, 43: 1940-1946

14 Alaee M, Arias P, Sjodin A, et al. An overview of commercially used brominated flame retardants, their applications, their use patterns in different countries/regions and possible modes of release. Environ Int, 2003, 29: 683-689

15 Sjodin A, Patterson D G, Bergman A. A review on human exposure to brominated flame retardants-particularly polybrominated diphenyl ethers. Environ Int, 2003, 29: 829-839

16 Barontini F, Cozzani V, Petarca L. Thermal stability and decomposition products of hexabromocyclododecane. Ind Eng Chem Res, 2001, 40: $3270-3280$

17 Larsen E R, Ecker E L. Thermal-stability of fire retardants.1. Hexabromocyclododecane (HBCD). J Fire Sci, 1986, 4: 261-275

18 Tomy G T, Halldorson T, Danell R, et al. Refinements to the diastereoisomer-specific method for the analysis of hexabromocyclododecane. Rapid Commun Mass Spectrom, 2005, 19: 2819-2826

19 Guerra P, Eljarrat E, Barcel D. Enantiomeric specific determination of hexabromocyclododecane by liquid chromatography-quadrupole linear ion trap mass spectrometry in sediment samples. J Chromatogr A, 2008, 1203: 81-87

20 Gao S, Wang J, Yu Z, et al. Hexabromocyclododecanes in Surface Soils from E-Waste Recycling Areas and Industrial Areas in South China: Concentrations, Diastereoisomer- and Enantiomer-Specific Profiles, and Inventory. Environ Sci Technol, 2011, 45: 2093-2099

21 Morris S, Bersuder P, Allchin C R, et al. Determination of the bro- minated flame retardant, hexabromocyclodocane, in sediments and biota by liquid chromatography-electrospray ionisation mass spectrometry. TrAC, Trends Anal Chem, 2006, 25: 343-349

22 Budakowski W, Tomy G. Congener-specific analysis of hexabromocyclododecane by high-performance liquid chromatography/electrospray tandem mass spectrometry. Rapid Commun Mass Spectrom, 2003, 17: 1399-1404

23 Suzuki S, Hasegawa A. Determination of hexabromocyclododecane diastereoisomers and tetrabromobisphenol a in water and sediment by liquid chromatography/mass spectrometry. Anal Sci, 2006, 22: 469-474

24 Frederiksen M, Vorkamp K, Bossi R, et al. Method development for simultaneous analysis of HBCD, TBBPA, and dimethyl-TBBPA in marine biota from Greenland and the Faroe Islands. Int J Environ Anal Chem, 2007, 87: 1095-1109

25 Berger U, Herzke D, Sandanger T M. Two trace analytical methods for determination of hydroxylated PCBs and other halogenated phenolic compounds in eggs from Norwegian birds of prey. Anal Chem, 2004, 76: 441-452

26 Cariou R, Antignac J P, Marchand P, et al. New multiresidue analytical method dedicated to trace level measurement of brominated flame retardants in human biological matrices. J Chromatogr A, 2005, 1100: $144-152$

27 Morris S, Allchin C R, Zegers B N, et al. Distributon and fate of HBCD and TBBPA brominated flame retardants in north sea estuaries and aquatic food webs. Environ Sci Technol, 2004, 38: 5497-5504

28 Zhao X, Zhang H, Fan J, et al. Dioxin-like compounds in sediments from the Daliao River estuary of Bohai Sea: Distribution and their influencing factors. Mar Pollut Bull, 2011, 62: 918-925

29 Shi Z. The detection and exposure assessment of hexabromocyclodedocane and tetrabromobisphenol $\mathrm{A}$ in food. Dissertation for the Doctoral Degree. Beijing: Chinese Center for Disease Control and Prevention, 2009

30 Xie Z, Ebinghaus R, Lohmann R, et al. Trace determination of the flame retardant tetrabromobisphenol $\mathrm{A}$ in the atmosphere by gas chromatography-mass spectrometry. Anal Chim Acta, 2007, 584: 333-342

31 De Boer J, Allchin C, Law R, et al. Method for the analysis of polybrominated diphenylethers in sediments and biota. TrAC, Trends Anal Chem, 2001, 20: 591-599

32 WHO. Tetrabromobisphenol A and derivatives. Environmental Health Criteria 172. 1995

33 Isabel Garcia-valcarcel A, Luis Tadeo J. Determination of hexabromocyclododecane isomers in sewage sludge by LC-MS/MS. J Sep Sci, 2009, 32: 3890-3897

34 Guo L, Ba T, Zheng M. Study of sources and distribution characteristics of polychlorinated naphthalenes. Prog Chem, 2009, 21: 377-388

Open Access This article is distributed under the terms of the Creative Commons Attribution License which permits any use, distribution, and reproduction in any medium, provided the original author(s) and source are credited. 